PDES, SUBMANIFOLDS AND

AFFINE DIFFERENTIAL GEOMETRY

BANACH CENTER PUBLICATIONS, VOLUME 57

INSTITUTE OF MATHEMATICS

POLISH ACADEMY OF SCIENCES

WARSZAWA 2002

\title{
A HOLOMORPHIC REPRESENTATION FORMULA FOR PARABOLIC HYPERSPHERES
}

\author{
VICENTE CORTÉS \\ Mathematisches Institut, Universität Bonn \\ Beringstraße 1, D-53115 Bonn, Germany \\ E-mail:vicente@math.uni-bonn.de
}

\begin{abstract}
A holomorphic representation formula for special parabolic hyperspheres is given.
Introduction. It was noticed by Blaschke that parabolic spheres in affine 3-space admit parametrisations in terms of holomorphic functions (of one variable). This is related to the fact that the Monge-Ampère equation governing parabolic hyperspheres is completely integrable in dimension two, a fact already known to Monge. The purpose of this note is to derive an explicit formula describing special parabolic hyperspheres in affine $(2 n+1)$-space in terms of a holomorphic function of $n$ variables.
\end{abstract}

1. Special parabolic affine hyperspheres. Let me briefly recall the notion of special parabolic affine hypersphere $[\mathrm{BC}]$. We consider $\mathbb{R}^{m+1}$ as affine space with standard connection denoted by $\widetilde{\nabla}$ and parallel volume form vol. A hypersurface is given by an immersion $\varphi: M \rightarrow \mathbb{R}^{m+1}$ of an $m$-dimensional connected manifold. We assume that $M$ admits a transversal vector field $\xi$ and that $m>1$. This induces on $M$ the volume form $\nu=\operatorname{vol}(\xi, \ldots)$, a torsionfree connection $\nabla$, a quadratic covariant tensor field $g$, an endomorphism field $S$ (shape tensor) and a one-form $\theta$ such that

$$
\widetilde{\nabla}_{X} Y=\nabla_{X} Y+g(X, Y) \xi, \quad \widetilde{\nabla}_{X} \xi=S X+\theta(X) \xi .
$$

Let us call the data $(\nabla, g, S, \theta)$ the Gauß-Weingarten data induced by the transversal vector field $\xi$. We will assume that $g$ is nondegenerate and, hence, is a pseudo-Riemannian metric on $M$. This condition does not depend on the choice of $\xi$. According to Blaschke [B], once the orientation of $M$ is fixed, there is a unique choice of transversal vector field $\xi$

2000 Mathematics Subject Classification: 53A15, 53C26.

Key words and phrases: affine hyperspheres, special Kähler manifolds.

This work was supported by SFB 256 "Nichtlineare partielle Differentialgleichungen" (Universität Bonn).

The paper is in final form and no version of it will be published elsewhere. 
such that $\nu$ coincides with the metric volume form vol ${ }^{g}$ and $\nabla \nu=0$. This particular choice of transversal vector field is called the affine normal and the corresponding geometric data $(g, \nabla)$ are called Blaschke metric and induced connection. Notice that, for the affine normal, $\theta=0$ and $S$ is computable from $(g, \nabla)$ (Gauß equations). Henceforth we use always the affine normal as transversal vector field.

Definition 1. The hypersurface $\varphi: M \rightarrow \mathbb{R}^{m+1}$ is called a parabolic (or improper) hypersphere if the affine normal is parallel, $\widetilde{\nabla} \xi=0$. It is called special if there exists an almost complex structure $J$ on $M$ which is skew symmetric with respect to the Blaschke metric $g$ and such that the 2 -form $\omega:=g(J \cdot, \cdot)$ is parallel with respect to the induced connection $\nabla$. Such an almost complex structure $J$ is called compatible.

Notice that $\widetilde{\nabla} \xi=0 \Leftrightarrow S=0 \Leftrightarrow \nabla$ is flat. It was proven in [BC] that any parabolic two-dimensional sphere with (positive or negative) definite Blaschke metric is special and that a compatible almost complex structure on a special parabolic hypersphere is necessarily integrable. In fact, we proved the following stronger result, Theorem 1 below.

Recall that a special Kähler manifold $(M, J, g, \nabla)$ is a (pseudo-)Kähler manifold $(M, J, g)$ endowed with a flat torsionfree connection $\nabla$ such that $\nabla J$ is symmetric and $\nabla \omega=0$, where $\omega=g(J \cdot, \cdot)$ is the Kähler form.

THEOREM $1[\mathrm{BC}]$. Let $\varphi: M \rightarrow \mathbb{R}^{m+1}$ be a special parabolic hypersphere with Blaschke metric $g$, induced connection $\nabla$, compatible almost complex structure $J$ and canonical two-form $\omega=g(J \cdot, \cdot)$. Then $(M, J, g, \nabla)$ is a special Kähler manifold. Conversely, any simply connected special Kähler manifold $(M, J, g, \nabla)$ admits an immersion $\varphi: M \rightarrow$ $\mathbb{R}^{m+1}$, which is a special parabolic hypersphere with Blaschke metric $g$, induced connection $\nabla$ and compatible almost complex structure $J$. The immersion $\varphi$ is unique up to a unimodular affine transformation of $\mathbb{R}^{m+1}$.

2. The holomorphic representation formula. It was proven in $[A C D]$ that any special Kähler manifold is locally defined by a holomorphic function, as follows. Let $F$ be a holomorphic function on a domain (i.e. a connected open set) $U \subset \mathbb{C}^{n}$ such that the (real) matrix

$$
\operatorname{Im} \partial^{2} F \quad \text { is invertible, }
$$

where $\partial^{2} F$ denotes the holomorphic Hessian of $F$. Let us denote by $M_{F} \subset T^{*} \mathbb{C}^{n}$ the image of the holomorphic section $d F: U \rightarrow T^{*} U \subset T^{*} \mathbb{C}^{n}$. It is a complex Lagrangian submanifold with respect to the standard complex symplectic structure $\Omega=\sum d z^{i} \wedge d w_{i}$, where $\left(z^{1}, \ldots, z^{n}, w_{1}, \ldots, w_{n}\right)$ are canonical coordinates of $T^{*} \mathbb{C}^{n}$. We denote its complex structure by $J$. Using the nondegeneracy condition $(2.1)$, it is shown in $[\mathrm{ACD}]$ that the Hermitian form $\gamma:=\sqrt{-1} \Omega(\cdot, \cdot)$ is nondegenerate on $M_{F}$ and, hence, induces a (pseudo-) Kähler metric $g=\left.\operatorname{Re} \gamma\right|_{M_{F}}$. It is also shown that a torsionfree connection $\nabla$ on $M_{F}$ can be defined by the condition that the real parts $x^{i}:=\operatorname{Re} z^{i}$ and $y_{j}:=\operatorname{Re} w_{j}$ are $\nabla$-affine functions on $M_{F}$. In fact, it is shown that $\left(x^{1}, \ldots, x^{n}, y_{1}, \ldots, y_{n}\right)$ is a (real) local coordinate system near any point of $M_{F}$ and that the Kähler form $\omega=g(\cdot, J \cdot)$ is expressed by the formula $\omega=2 \sum d x^{i} \wedge d y_{i}$ on $M_{F}$. 
THEOREM $2[\mathrm{ACD}]$. Let $F$ be a holomorphic function satisfying the nondegeneracy condition (2.1) on a domain $U \subset \mathbb{C}^{n}$. Then $\left(M_{F}, J, g, \nabla\right)$, defined above, is a special Kähler manifold and any special Kähler manifold is locally of this form.

It is noticed in $[\mathrm{BC}]$ that combining Theorem 1 and Theorem 2 we can associate a parabolic hypersphere to any holomorphic function $F$ defined on a simply connected domain $U \subset \mathbb{C}^{n}$ and satisfying the nondegeneracy condition (2.1). However, the proof of Theorem 1 makes use of the Fundamental Theorem of affine differential geometry [DNV] (the generalisation of Radon's theorem [R] to higher dimensions) and does not involve any explicit parametrisation of the immersion $\varphi: M \rightarrow \mathbb{R}^{2 n+1}$ realising a simply connected special Kähler manifold $(M, J, g, \nabla)$ of real dimension $2 n$ as parabolic hypersphere. The aim is now to provide an explicit formula, in terms of the holomorphic function $F$, for the realisation of $\left(M_{F}, J, g, \nabla\right)$ as a parabolic hypersphere $\varphi_{F}: M_{F} \cong U \rightarrow \mathbb{R}^{2 n+1}$.

We will not restrict ourselves to functions $F$ defined on simply connected domains $U \subset \mathbb{C}^{n}$. More generally, we consider a 'multivalued' function defined on an arbitrary domain $U \subset \mathbb{C}^{n}$. Or, in other words, a (univalued) function defined on some Riemann domain $\widetilde{U}$ over $U$. A Riemann domain over $U$ is a holomorphic (unramified) covering $\pi$ : $\widetilde{U} \rightarrow U$. Any holomorphic function $F$ on $\widetilde{U}$ defines a holomorphic Lagrangian immersion

$$
\phi: \widetilde{U} \rightarrow T^{*} U \subset T^{*} \mathbb{C}^{n}, \quad \phi(p):=d F \circ\left(\pi_{*} \mid T_{p} \widetilde{U}\right)^{-1}, \quad p \in \widetilde{U} .
$$

Let us denote by $J$ the complex structure of $\widetilde{U}$. Pulling back the canonical coordinates of $T^{*} \mathbb{C}^{n}$ to $\widetilde{U}$ we obtain holomorphic functions

$$
\tilde{z}^{i}:=\phi^{*} z^{i} \quad \text { and } \quad \tilde{w}_{j}:=\phi^{*} w_{j}
$$

on $\widetilde{U}$. The holomorphic functions $\tilde{z}^{i}$ form a local holomorphic coordinate system near any point of $\widetilde{U}$. We use the compact notation

$$
\tilde{z}:=\left(\tilde{z}^{1}, \ldots, \tilde{z}^{n}\right), \quad F_{\tilde{z}}=\left(F_{\tilde{z}^{1}}, \ldots, F_{\tilde{z}^{n}}\right)=\left(\frac{\partial F}{\partial \tilde{z}^{1}}, \ldots, \frac{\partial F}{\partial \tilde{z}^{n}}\right), \quad F_{\tilde{z}} \tilde{z}=\sum F_{\tilde{z}^{k}} \tilde{z}^{k} \quad \text { etc. }
$$

Let $\partial^{2} F$ be the Hessian of $F$ with respect to (the flat torsionfree holomorphic connection defined by) the coordinate system $\tilde{z}$. We call $F$ nondegenerate if $\operatorname{Im} \partial^{2} F$ is invertible. Then, as before, $g:=\operatorname{Re} \phi^{*} \gamma$ is a pseudo-Kähler metric and we can define a flat torsionfree connection $\nabla$ by the condition that the functions $\tilde{x}^{i}:=\operatorname{Re} \tilde{z}^{i}$ and $\tilde{y}_{j}:=\operatorname{Re} \tilde{w}_{j}$ are $\nabla$-affine functions on $\widetilde{U}$. We also put $\tilde{u}^{i}:=\operatorname{Im} \tilde{z}^{i}$ and $\tilde{v}_{j}:=\operatorname{Im} \tilde{w}_{j}$. Let us abbreviate $M(F):=(\widetilde{U}, J, g, \nabla)$ and define an immersion $\varphi_{F}: \widetilde{U} \rightarrow \mathbb{R}^{2 n+1}$ by the formula

$$
\begin{aligned}
\varphi_{F} & :=\left(\operatorname{Re} \tilde{z}, \operatorname{Re} F_{\tilde{z}}, 2 \operatorname{Im} F-2\left(\operatorname{Re} F_{\tilde{z}}\right) \operatorname{Im} \tilde{z}\right) \\
& =\left(\tilde{x}^{1}, \ldots, \tilde{x}^{n}, \tilde{y}_{1}, \ldots, \tilde{y}_{n}, 2 \operatorname{Im} F-2 \sum\left(\tilde{y}_{k}\right) \tilde{u}^{k}\right) .
\end{aligned}
$$

THEOREM 3. Let $F$ be a nondegenerate holomorphic function defined on a Riemann domain $\widetilde{U}$. Then $M(F)=(\widetilde{U}, J, g, \nabla)$, defined above, is a special Kähler manifold with Kähler form $\omega=g(\cdot, J \cdot)=2 \sum d \tilde{x}^{i} \wedge d \tilde{y}_{i}$. The immersion $\varphi_{F}: \widetilde{U} \rightarrow \mathbb{R}^{2 n+1}$ defined by (2.3) is, with respect to the volume form $\mathrm{vol}:=2^{n} \operatorname{det}$ on $\mathbb{R}^{2 n+1}$, a special parabolic hypersphere with affine normal $\xi=\partial_{2 n+1}$, Blaschke metric $g$, induced connection $\nabla$ and compatible almost complex structure $J$. It is unique up to unimodular affine transformations of $\mathbb{R}^{2 n+1}$. 
Proof. The first statement is a slight generalisation of the first part of Theorem 2, with essentially the same proof. The uniqueness of $\varphi_{F}$ follows, as in the proof of Theorem 1, from the uniqueness statement of the Fundamental Theorem of affine differential geometry. It suffices to prove that $\varphi_{F}$ is a parabolic hypersphere with Blaschke metric $g$, induced connection $\nabla$ and compatible almost complex structure $J$. Let us compute the Gauß-Weingarten data $\left(\nabla^{v}, g^{v}, S^{v}, \theta^{v}\right)$, see (1.1), induced by the transversal vector field $v=\partial_{2 n+1}$ (the 'vertical' vector field). It is immediate that $S^{v}=0$ and $\theta^{v}=0$. We compute $\nabla^{v}$ and $g^{v}$ for the coordinate vector fields

$$
\partial_{\tilde{x}^{i}}=\partial_{i}+\frac{\partial f}{\partial \tilde{x}^{i}} \partial_{2 n+1}, \quad \partial_{\tilde{y}_{j}}=\partial_{n+j}+\frac{\partial f}{\partial \tilde{y}_{j}} \partial_{2 n+1},
$$

where

$$
f:=2 \operatorname{Im} F-2\left(\operatorname{Re} F_{\tilde{z}}\right) \operatorname{Im} \tilde{z}=2 \operatorname{Im} F-2 \sum \tilde{y}_{k} \tilde{u}^{k}
$$

is the last component of $\varphi_{F}$. The covariant derivatives with respect to the connection $\widetilde{\nabla}$ of $\mathbb{R}^{2 n+1}$ are given by

$$
\widetilde{\nabla}_{\partial_{\tilde{x}^{i}}} \partial_{\tilde{x}^{j}}=\frac{\partial^{2} f}{\partial_{\tilde{x}^{i}} \partial_{\tilde{x}^{j}}} v, \quad \widetilde{\nabla}_{\partial_{\tilde{x}^{i}}} \partial_{\tilde{y}_{j}}=\widetilde{\nabla}_{\partial_{\tilde{y}_{j}}} \partial_{\tilde{x}^{i}}=\frac{\partial^{2} f}{\partial_{\tilde{x}^{i}} \partial_{\tilde{y}_{j}}} v, \quad \widetilde{\nabla}_{\partial_{\tilde{y}_{i}}} \partial_{\tilde{y}_{j}}=\frac{\partial^{2} f}{\partial_{\tilde{y}_{i}} \partial_{\tilde{y}_{j}}} v .
$$

This shows that the coordinate vector fields $\partial_{\tilde{x}^{i}}$ and $\partial_{\tilde{y}_{j}}$ are parallel for the connection $\nabla^{v}$, so it coincides with $\nabla$. Now $\theta=0$ implies $\nabla \nu^{v}=\nabla^{v} \nu^{v}=0$ for the volume form $\nu^{v}=\operatorname{vol}(v, \ldots)$. Moreover, we see that $g^{v}=\operatorname{Hess}^{\nabla}(f)=\nabla^{2} f$.

Claim 1. $g^{v}=g$.

The claim, to be proven below, implies that $v$ is the affine normal and, hence, that $g^{v}=g$ is the Blaschke metric. Let us see why. The Riemannian volume of the (pseudo-) Kähler manifold $M(F)$ with Kähler form $\omega=g(J \cdot, \cdot)=2 \sum_{i=1}^{n} d \tilde{x}^{i} \wedge d \tilde{y}_{i}$ is given by

$$
\mathrm{vol}^{g}=(-1)^{n(n-1) / 2} \frac{\omega^{n}}{n !}=2^{n} d \tilde{x}^{1} \wedge \ldots \wedge d \tilde{x}^{n} \wedge d \tilde{y}_{1} \wedge \ldots \wedge d \tilde{y}_{n}=2^{n} \operatorname{det}(v, \ldots)=\nu^{v}
$$

if we choose the orientation defined by $\nu^{v}$. (Notice that $d \tilde{x}^{1} \wedge \ldots \wedge d \tilde{x}^{n} \wedge d \tilde{y}_{1} \wedge \ldots \wedge d \tilde{y}_{n}=$ $(-1)^{n(n-1) / 2} d \tilde{x}^{1} \wedge d \tilde{y}_{1} \wedge \ldots \wedge d \tilde{x}^{n} \wedge d \tilde{y}_{n}$.) This shows that vol ${ }^{g}$ coincides with the $\nabla$-parallel volume form $\nu^{v}$. So $v$ is the affine normal and, hence, $\varphi_{F}$ is a parabolic hypersphere with Blaschke metric $g$ and induced connection $\nabla$. The fact that $M(F)$ is a special Kähler manifold entails that $J$ is skew symmetric with respect to $g$ and $\nabla \omega=0$. Therefore, the complex structure $J$ is compatible, in the sense of Definition 1 , with the data $(g, \nabla)$.

It remains to prove Claim 1. For the calculations we will use the next lemma.

Lemma 1. The partial derivatives of the functions $\tilde{u}^{i}$ and $\tilde{v}_{j}$ on $M(F)$ with respect to the $\nabla$-affine coordinates $\left(\tilde{x}^{1}, \ldots, \tilde{x}^{n}, \tilde{y}_{1}, \ldots, \tilde{y}_{n}\right)$ satisfy the following equations:

$$
\begin{gathered}
\sum_{k}\left(\tilde{u}_{\tilde{x}^{i}}^{k}\left(\tilde{v}_{k}\right)_{\tilde{y}_{j}}-\tilde{u}_{\tilde{y}_{j}}^{k}\left(\tilde{v}_{k}\right)_{\tilde{x}^{i}}\right)=\delta_{i}^{j} \\
\sum_{k} \tilde{u}_{\tilde{x}^{i}}^{k}\left(\tilde{v}_{k}\right)_{\tilde{x}^{j}}=\sum_{k} \tilde{u}_{\tilde{x}^{j}}^{k}\left(\tilde{v}_{k}\right)_{\tilde{x}^{i}}, \quad \sum_{k} \tilde{u}_{\tilde{y}_{i}}^{k}\left(\tilde{v}_{k}\right)_{\tilde{y}_{j}}=\sum_{k} \tilde{u}_{\tilde{y}_{j}}^{k}\left(\tilde{v}_{k}\right)_{\tilde{y}_{i}} \\
\tilde{u}_{\tilde{x}^{j}}^{i}=-\left(\tilde{v}_{j}\right)_{\tilde{y}_{i}}, \quad \tilde{u}_{\tilde{y}_{j}}^{i}=\tilde{u}_{\tilde{y}_{i}}^{j}, \quad\left(\tilde{v}_{i}\right)_{\tilde{x}^{j}}=\left(\tilde{v}_{j}\right)_{\tilde{x}^{i}} .
\end{gathered}
$$


Proof. Pulling back the symplectic form $\Omega$ of $T^{*} \mathbb{C}^{n}$ by means of the Lagrangian immersion $\phi: M(F) \rightarrow T^{*} \mathbb{C}^{n}$ defined in (2.2), we obtain the equation $\phi^{*} \Omega=0$. Decomposing it into real and imaginary parts yields the lemma.

Let us return to the proof of Theorem 3. First we observe that $\partial_{\tilde{x}^{i}} \operatorname{Im} F=\operatorname{Im} \partial_{\tilde{x}^{i}} F=\sum_{j} \operatorname{Im}\left(\frac{\partial \tilde{z}^{j}}{\partial \tilde{x}^{i}} \partial_{\tilde{z}^{j}} F\right)=\sum_{j} \operatorname{Im}\left(\left(\delta_{i}^{j}+\sqrt{-1} \tilde{u}_{\tilde{x}^{i}}^{j}\right) F_{\tilde{z}^{j}}\right)=\tilde{v}_{i}+\sum_{j} \tilde{u}_{\tilde{x}^{i}}^{j} \tilde{y}_{j}$ and

$$
\partial_{\tilde{y}_{j}} \operatorname{Im} F=\operatorname{Im} \partial_{\tilde{y}_{j}} F=\sum_{k} \operatorname{Im}\left(\frac{\partial \tilde{z}^{k}}{\partial \tilde{y}_{j}} \partial_{\tilde{z}^{k}} F\right)=\sum_{k} \operatorname{Im}\left(\sqrt{-1} \tilde{u}_{\tilde{y}_{j}}^{k} F_{\tilde{z}_{k}}\right)=\sum_{k} \tilde{u}_{\tilde{y}_{j}}^{k} \tilde{y}_{k} .
$$

The second derivatives of $\operatorname{Im} F$ are now easily computed with the help of Lemma 1:

$\partial_{\tilde{x}^{i} \tilde{x}^{j}}^{2} \operatorname{Im} F=\left(\tilde{v}_{i}\right)_{\tilde{x}^{j}}+\sum_{k} \tilde{u}_{\tilde{x}^{i} \tilde{x}^{j}}^{k} \tilde{y}_{k}, \partial_{\tilde{x}^{i} \tilde{y}_{j}}^{2} \operatorname{Im} F=\sum_{k} \tilde{u}_{\tilde{y}_{j} \tilde{x}^{i}}^{k} \tilde{y}_{k}, \partial_{\tilde{y}_{i} \tilde{y}_{j}}^{2} \operatorname{Im} F=\tilde{u}_{\tilde{y}_{j}}^{i}+\sum_{k} \tilde{u}_{\tilde{y}_{i} \tilde{y}_{j}}^{k} \tilde{y}_{k}$.

Using this and Lemma 1 one can now evaluate $g^{v}=\nabla^{2} f$ :

$$
\begin{aligned}
& g^{v}\left(\partial_{\tilde{x}^{i}}, \partial_{\tilde{x}^{j}}\right)=\partial_{\tilde{x}^{i} \tilde{x}^{j}}^{2} f=2\left(\left(\tilde{v}_{i}\right)_{\tilde{x}^{j}}+\sum_{k} \tilde{u}_{\tilde{x}^{i} \tilde{x}^{j}}^{k} \tilde{y}_{k}\right)-2 \sum_{k} \tilde{u}_{\tilde{x}^{i} \tilde{x}^{j}}^{k} \tilde{y}_{k}=2\left(\tilde{v}_{i}\right)_{\tilde{x}^{j}}, \\
& g^{v}\left(\partial_{\tilde{x}^{i}}, \partial_{\tilde{y}_{j}}\right)=\partial_{\tilde{x}^{i} \tilde{y}_{j}}^{2} f=2 \sum_{k} \tilde{u}_{\tilde{y}_{j} \tilde{x}^{i}}^{k} \tilde{y}_{k}-2\left(\tilde{u}_{\tilde{x}^{i}}^{j}+\sum_{k} \tilde{u}_{\tilde{y}_{j} \tilde{x}^{i}}^{k} \tilde{y}_{k}\right)=-2 \tilde{u}_{\tilde{x}^{i}}^{j}, \\
& g^{v}\left(\partial_{\tilde{y}_{i}}, \partial_{\tilde{y}_{j}}\right)=\partial_{\tilde{y}_{i} \tilde{y}_{j}}^{2} f=2\left(\tilde{u}_{\tilde{y}_{j}}^{i}+\sum_{k} \tilde{u}_{\tilde{y}_{i} \tilde{y}_{j}}^{k} \tilde{y}_{k}\right)-2\left(\tilde{u}_{\tilde{y}_{i}}^{j}+\tilde{u}_{\tilde{y}_{j}}^{i}+\sum_{k} \tilde{u}_{\tilde{y}_{i} \tilde{y}_{j}}^{k} \tilde{y}_{k}\right)=-2 \tilde{u}_{\tilde{y}_{j}}^{i} .
\end{aligned}
$$

Notice that in virtue of $(2.4)$ we have:

$$
\left(\tilde{u}^{i}\right)_{\tilde{x}^{j}}=\left(\tilde{u}^{j}\right)_{\tilde{x}^{i}}
$$

Let us compare this with $g$. The simplest way to compute $g$ is using the fact that $g=\omega \circ J$, where $\omega=2 \sum d x^{i} \wedge d y_{i}$ is the Kähler form and we consider $g$ and $\omega$ as isomorphisms $T M \rightarrow T^{*} M$ (insertion of a vector in the first argument). It is easier to work with the inverse metric $g^{-1}=J^{-1} \circ \omega^{-1}=-J \circ \omega^{-1}=\omega^{-1} \circ J^{*}$. Notice that

$$
\omega^{-1}=\frac{1}{2} \sum \partial_{\tilde{y}_{i}} \wedge \partial_{\tilde{x}^{i}}, \quad J^{*} d \tilde{x}^{i}=-d \tilde{u}^{i} \quad \text { and } \quad J^{*} d \tilde{y}_{j}=-d \tilde{v}_{j}
$$

Let us evaluate $g^{-1}$ with the help of these formulas and Lemma

$$
\begin{aligned}
& g^{-1}\left(d \tilde{x}^{i}, d \tilde{x}^{j}\right)=-\omega^{-1}\left(d \tilde{u}^{i}, d \tilde{x}^{j}\right)=-\frac{1}{2} \tilde{u}_{\tilde{y}_{j}}^{i}, \\
& g^{-1}\left(d \tilde{x}^{i}, d \tilde{y}_{j}\right)=-\omega^{-1}\left(d \tilde{u}^{i}, d \tilde{y}_{j}\right)=\frac{1}{2} \tilde{u}_{\tilde{x}^{j}}^{i}, \\
& g^{-1}\left(d \tilde{y}_{i}, d \tilde{y}_{j}\right)=-\omega^{-1}\left(d \tilde{v}_{i}, d \tilde{y}_{j}\right)=\frac{1}{2}\left(\tilde{v}_{i}\right)_{\tilde{x}^{j}} .
\end{aligned}
$$

Comparing with the formulas for $g^{v}$ and using Lemma 1 and (2.5) this proves that $g^{-1} g^{v}=\mathrm{id}$ and, hence, that $g=g^{v}$. This completes the proof of Claim 1 and Theorem 3 .

\section{References}

[ACD] D. V. Alekseevsky, V. Cortés and C. Devchand, Special complex manifolds, J. Geom. Phys. 42 (2002), 85-105. 
[BC] O. Baues and V. Cortés, Realisation of special Kähler manifolds as parabolic spheres, Proc. Amer. Math. Soc. 129 (2001), 2403-2407.

[B] W. Blaschke, Vorlesungen über Differentialgeometrie II. Affine Differentialgeometrie, Grundlehren der Mathematischen Wissenschaften VII, Springer, Berlin, 1923.

[DNV] F. Dillen, K. Nomizu and L. Vrancken, Conjugate connections and Radon's theorem in affine differential geometry, Monatsh. Math. 109 (1990), 221-235.

[R] J. Radon, Die Grundgleichungen der affinen Flächentheorie, Leipziger Berichte 70 (1918), 91-107. 